\title{
Justicia distributiva, los trasplantes y la pandemia por SARS-CoV-2
}

\section{Distributive justice, transplantation and the SARS-CoV-2 pandemic}

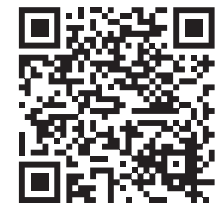

\author{
Guillermo Cantú-Quintanilla, ${ }^{*}$ Josefina Alberú, ${ }^{\ddagger}$ Rafael Reyes-Acevedo, ${ }^{\S}$ \\ Georgina Magaña," Silvia Chavira," Mara Medeiros** \\ * Departamento de Bioética de la Universidad Panamericana. Ciudad de México, México. \\ ₹ Tecnológico de Monterrey, Escuela de Medicina y Ciencias de la Salud. Monterrey, Nuevo León, México. \\ $\S$ Departamento de Trasplantes. Centenario Hospital Miguel Hidalgo. Aguascalientes, México. \\ " Unidad de Terapia Intensiva del Instituto Nacional de Neurología y Neurocirugía «Manuel Velasco Suárez». \\ Unidad de Cuidados Intensivos de Adultos, Hospital de Gineco-Obstetricia Núm. 4, IMSS. Ciudad de México, México. \\ " Asociación Mitigare Cuidados Paliativos A.C., San Miguel de Allende, Guanajuato, México. \\ ** Unidad de Investigación y Diagnóstico en Nefrología y Metabolismo Mineral Óseo del Hospital Infantil de México \\ «Federico Gómez». Departamento de Farmacología de la Facultad de Medicina, UNAM. Ciudad de México, México.
}

\section{RESUMEN}

La pandemia por SARS-CoV-2 ha tenido muchas implicaciones bioéticas para los pacientes con trasplante renal, un área en la que, sin duda, ya se tiene experiencia en la distribución de recursos escasos. Se discute el concepto de derecho y justicia distributiva, los cuales se abordan desde el contexto de la pandemia por SARS-CoV-2, el triage de pacientes con COVID-19 en hospitales sobrecargados, la necesidad de contar con cuidados paliativos y la reconversión de hospitales que anteriormente eran centros de trasplante.

Palabras clave: Justicia distributiva, SARS-CoV-2, COVID-19, trasplante.

\section{INTRODUCCIÓN}

Qué complejos resultan, en tiempos de pandemia, la identificación de los argumentos humanamente más sólidos para la distribución de la escasez.

Esta frase parte intencionadamente como introductoria por todo el acervo de preceptos ético-filosóficos que han tenido que salir nuevamente $-\mathrm{y}$ de manera álgida - a contribuir al pensamiento de quienes tienen el compromiso y la responsabilidad de identificar todos los elementos sustantivos en la aplicación de recur-

\section{ABSTRACT}

The SARS-CoV-2 pandemic has aroused several bioethics considerations for renal transplant recipients, an area that already has experience with scarce resource distribution. The concepts of right and distributive justice are discussed in the context of the SARS-CoV-2 pandemic situation, as well the COVID-19 patient triage in overwhelmed and saturated hospitals, the consequences of reconversion of hospital that were previously transplant centers and the growing need of palliative care.

Keywords: Distributive justice, SARS-CoV-2, COVID-19, transplantation.

sos, por demás escasos, para la atención de pacientes afectados por COVID-19, la enfermedad causada por el nuevo coronavirus SARS-CoV-2. En este sentido, vale repasar algunos conceptos, de suyo indispensables, para el mejor entendimiento de lo complejo de tal labor.

Si el ser humano se detiene a pensar qué es lo que más le indigna de las cosas que suceden a su alrededor, diría, tal vez, que la falta de libertad y de justicia. Cuesta trabajo dar con un concepto claro, unívoco e indiscutible de qué es la justicia a lo largo de los tiem- 
pos. Una posible causa de esa confusión estaría en pensar que la justicia se daría con independencia a la voluntad de los hombres. Por eso, es necesario considerar la justicia como un hábito virtuoso. Hacer justicia o administrar justicia es obligar a alguien a que dé, a la fuerza, lo que no quiso dar voluntariamente por virtud. La casi totalidad de la doctrina de la justicia residía ya en los pensadores griegos y en los juristas romanos donde nació la cultura occidental: Platón, Aristóteles, Cicerón, Ulpiano, Gayo, etcétera.

Hábito es aquella disposición estable que lleva a obrar; por eso se dice que hábitos operativos buenos o virtudes, son aquellos que perfeccionan la naturaleza humana. El hábito de la justicia es el derecho y el derecho es el objeto de la justicia.

El derecho objetivo es la cosa justa; una operación o realidad exterior, se habla, así, de precio justo, de salario justo.

El derecho subjetivo es la posibilidad moral de hacer o de omitir algo. Tenemos así el derecho a la vida, a la libertad de expresión, etc. A este derecho corresponde por parte de los demás un deber: el deber de reconocer ese derecho.

El derecho prescriptivo es el contenido de las leyes de una sociedad, su ordenamiento jurídico.

El derecho subjetivo se basa en el derecho objetivo, por eso éste es el más importante. El derecho es lo que es debido a alguien según una relación de igualdad, en virtud de la cual un objeto está ordenado a una persona, y se convierte en suyo propio.

El derecho es algo objetivo que está por encima de las diferencias personales o de las disposiciones del sujeto. El derecho, al ser objetivo, exige siempre una relación con el otro. De ahí la importancia social del derecho y de la justicia, cuyo acto final es el derecho.

Las relaciones sociales son, en casi todos los casos, relaciones jurídicas, relaciones de derecho y, finalmente, la implicatura de una relación de igualdad. Dar lo que es debido a otro significa establecer una igualdad entre lo que por justicia se tenga y lo que sólo se tiene.

Así entendemos que las propiedades de la justicia son la alteridad, pues se dirige al otro dar lo debido y dar hasta la igualdad. Hoy se hace el mejor esfuerzo para reconocer al otro como igual a los demás, entender qué se le debe y quién se lo da o reconoce.

La ley es la regla del derecho - la causa- porque, cuando hay ley, hay derecho. Si el objeto del derecho es lo debido al otro, esto se ha de ver reflejado en la ley. Por eso, según las clases de leyes, serán las clases de derechos. Se puede hablar de derecho na- tural porque hay ley natural, es decir, hacer el bien, no hacer daño, no hacer a otro lo que no quieres que te hagan, hacer el bien sin mirar a quién, sin hacer acepción de personas.

Pero no basta la ley natural para regir el entramado social, surge la ley positiva de la que emana el derecho positivo; ese objetivo de moderar y orientar las pretensiones de unos y otros que viven en comunidad. Entonces, ¿qué es lo debido al otro?

Lo entendemos de dos maneras, porque lo marca la misma naturaleza humana al tener una racionalidad libre, una inteligencia creativa. Así, todos por naturaleza son iguales, todos tienen vida, tienen necesidades materiales y humanas, por ejemplo, la alimentación, la educación en valores, etc. Luego, lo debido naturalmente se llama derecho natural: a la vida, el alimento, a la educación, la salud, etc. Pero también lo debido lo establece un acuerdo entre los ciudadanos. Ese acuerdo se positiviza, se plasma de forma neta en la ley positiva que dicta toda autoridad. El conjunto de esas leyes positivas es el derecho positivo.

Surge entonces las preguntas: ¿se puede garantizar el derecho a la salud?, ¿qué desigualdades en el acceso a la salud son injustas?

Primero, hace falta reconocer las cualidades de la ley positiva y sus consecuencias. Ha de ser legítima, procedente de la legítima autoridad, ha de ser racional, ha de estar requerida por la misma autoridad social competente, ha de tender a realizar el bien común y ha de ser suficientemente promulgada para que sea conocida. Con esas características, la ley positiva es justa y se convierte en una prolongación de la ley natural en su aplicación en las concretas circunstancias de lugar y tiempo.

Los derechos naturales son inherentes al hombre por el mero hecho de ser humano. Todos tienen derecho a la vida, a la integridad física, al alimento, a la educación, la salud, etc. Éstos los llamamos derechos fundamentales o simplemente derechos humanos. Sin embargo, para algunas ideologías, todos los derechos son positivos, es decir, producto de un acuerdo, consentimiento o consenso entre los hombres. En esas condiciones es el estado quien confiere esos derechos, por no reconocer y aceptar una naturaleza humana que los tiene como propios y los exige.

Los argumentos que se esgrimen con más frecuencia son que no es posible saber en qué consiste la naturaleza humana y, mucho menos, dilucidar si es algo estable o mudable. Se trata además de un supuesto derecho natural que no está vigente por sí solo. Y, por si fuera poco, los llamados «derechos naturales» han 
sido negados a lo largo de la historia y han sido también incumplidos sin que sucediese nada especial. ${ }^{1}$

En contraparte a los argumentos anteriores, se podría decir que es posible saber, racionalmente y de un modo simplemente lógico, qué es la naturaleza humana, la persona como ser racional y libre, con una serie de exigencias naturales: la vida, la alimentación, la vivienda, la salud, el trabajo, la seguridad, etc., que se ha dado en todos los tiempos y se sigue dando.

La vigencia del derecho natural está clara, pero como una realidad moral, es decir, como algo dirigido a la libertad, algo que el hombre debe cumplir, aunque no le guste.

El incumplimiento del derecho natural no quiere decir que no exista o que no esté vigente. Además, los principales preceptos del derecho natural han sido recogidos en el derecho positivo, pero no todos ni siempre, como lo observamos en la actualidad. Por ejemplo, si un Estado ordenase, como ley, la muerte de los ancianos, esa ley sería injusta, no habría obligación moral de cumplirla, más bien habría obligación moral de no cumplirla. ¿Por qué existen derechos naturales, fundamentales, elementales, inalienables, humanos? ¿Por qué tengo que dar naturalmente lo debido y propio del otro?

Si no podemos argumentar que actuar de forma contraria niega y destroza la convivencia social, no sería posible nunca protestar o reclamar los abusos e injusticias actuales.

¿Por qué tengo que dar al otro lo suyo? La respuesta es: porque es suyo y no mío. Si es suyo, ¿cómo lo ha conseguido?, ¿cómo lo ha obtenido? No por acuerdo de la sociedad o del Estado, porque si fuera así, la sociedad y el Estado se lo podrían quitar en cualquier momento o incluso declarar, con una ley, que ya no hay, de ahora en adelante, derecho a la vida, o a la información, o a la libertad de expresión o a la educación, etc.

Para algunos, el ser humano es un animal evolucionado, un individuo que, por azar, ha llegado a ser lo que es $y$, al darse cuenta, a diferencia de los otros animales, que es útil y conveniente, hablar de derechos humanos, para que pueda haber un cierto orden en la sociedad.

Para otros existe un orden superior y trascendente que impone el deber de respetar los derechos de los demás seres humanos, de reconocer lo que es debido a su naturaleza que conoce su fin y delibera sobre los medios para conseguirlo.

La segunda postura permite denunciar las situaciones de tiranía, de opresión y de injusticia que pueden ser impuestas por un régimen totalitario, que conside- ra que los derechos los otorga la sociedad o cualquier otra estructura suya.

El orden de la ley natural y del derecho natural es un orden ético o moral. Ese orden se refiere a todos los actos humanos, externos e internos, y el orden jurídico sólo a los que hacen relación con lo otro, es decir, a los actos externos. Por eso, todos los actos jurídicos son éticos, buenos o malos, pero no todos los actos morales son jurídicos.

Un orden jurídico tiene que ser moralmente correcto. Cuando no es así, es probable que en la sociedad se produzcan conflictos. Citemos sólo el caso de que algunos ciudadanos se niegan, a conciencia, a cumplir una ley que es injusta, porque va en contra del orden moral, que es objetivo y natural.

El derecho se ha limitado, en una medida creciente, a castigar como delitos sólo aquéllos que podrían causar un daño a terceros, a la seguridad pública o al ordenamiento del Estado.

En regímenes totalitarios o de partido único, la decisión sobre lo que es inmoral o ilegal, o legal e inmoral a la vez, depende de pocas personas, de los que detentan el poder. Los ciudadanos pueden intervenir activamente no sólo a través del voto, sino de un comportamiento basado en la observancia de la ley natural. Es posible también que la mayoría de los ciudadanos presionen legítimamente a favor de soluciones auténticamente éticas.

Las consideraciones previas sobre la justicia, la libertad, el derecho, la naturaleza humana y la ley y sus divisiones nos servirán para entender la situación que ha generado la pandemia en nuestro medio y sus implicaciones en las políticas de salud, como se verá a continuación.

\section{LA JUSTICIA DISTRIBUTIVA}

Algunas veces se hace una distinción entre la justicia distributiva y la correctiva (conmutativa o rectificadora). La primera tiene que ver con la distribución de los beneficios y las cargas en ausencia de maldad anterior, mientras que la otra está relacionada con el modo de responder a la maldad pasada, como el castigo y la recompensa. Las políticas de salud de un país son una aplicación concreta de las nociones señaladas. Un ejemplo sería no atender adecuadamente los programas de educación y prevención en salud con su impacto en los costos por el tratamiento o rehabilitación en esas mismas enfermedades. ${ }^{2}$

Se puede hacer también una distinción entre la justicia ideal y la justicia práctica. La justicia ideal o plena 
es la que no cuenta con ninguna restricción empírica, como sería tener los recursos limitados; mientras que la justicia práctica se enfoca sobre los medios disponibles y requiere un juego factible de opciones, lo que resulta de particular relevancia para este tema, no parece haber solución única razonable para la pobreza o el desempleo ni en los países más desarrollados.

La justicia es típicamente analizada como permitir algunas cosas y prohibir otras, pero a veces también se analiza de manera axiológica, como sostener que algunas cosas son más justas que otras. Esta última versión nos hace aspirar y querer alcanzar de manera gradual lo que resulta mejor.

Un punto de vista es que la moneda de la justicia es el bienestar, visto tanto objetivo (el acceso a una alimentación, vivienda y educación) como a través de la percepción subjetiva de lo mismo (calidad de vida). El bienestar puede ser interpretado como felicidad, satisfacción de preferencias y, para algunos autores, necesidades más específicas, por ejemplo, el conocimiento o la amistad.

Supongamos que cada uno comienza con un bienestar igual y oportunidades efectivamente iguales, lo que se consideraría justo. El individuo debe establecer una reciprocidad y contribuir a multiplicar los bienes recibidos. Esto es el mínimo necesario para llegar a ser ciudadano de la época y terminar por obtener un nivel de vida adecuado.

\section{PATRONES DE DISTRIBUCIÓN DE BIENES ESCASOS: IGUALDAD, PRIORIDAD, SUFICIENCIA Y MERECIMIENTO}

Aquí examinaremos brevemente cuatro de los principales patrones de distribución invocados por la teoría de la justicia. Aunque cada uno puede ser presentado como parte de una teoría deontológica - de los deberes-, se verá si sus consecuencias maximizan la igualdad de resultados.

Los igualitaristas sostienen que la justicia se preocupa por la igualdad de algunos beneficios relevantes. ${ }^{3}$ El igualitarismo es una teoría comparativa: sólo se preocupa de la manera en que los beneficios de una persona se comparan con los de otros..$^{3,4}$ Como una teoría de la justicia comparativa, es muy loable en contextos en donde a cada uno se le debe el mismo nivel de beneficios, considerando los mínimos ya señalados de las dos dimensiones del bienestar. Sin embargo, hoy nadie defiende al igualitarismo puro como una teoría de la justicia que se pueda implementar.

Una forma diferente de distribución de bienes que tiene una especial preocupación por aquellos que ca- recen de lo necesario y viven desfavorecidos en la sociedad se conoce como el prioritarismo. ${ }^{5}$ Esta postura señala la mayor importancia moral de obtener los beneficios especificados para aquellos que tienen menos. El incrementar los beneficios en una unidad para los pobres es más relevante que el hacerlo para los prósperos. El principio de la diferencia o maximin significa maximizar el mínimo léxicamente para que la peor posición de prosperidad pueda llevarse a la mejor posición que sea posible. En caso de imposibilidad, la segunda peor posición deberá llevarse a la mejor posición posible, y así para la tercera, cuarta, etcétera.

El maximin concede un peso infinitamente mayor a los beneficios de una persona menos favorecida. Sostiene que dar cualquier beneficio (por pequeño que este sea) a una persona poco favorecida es mejor que dar un beneficio (por grande que este sea) a una persona más próspera. La equidad que surge es una especie de reparación de la injusticia inicial.

Mientras que el igualitarismo se preocupa del interés comparativo de dar a la gente en partes iguales y el prioritarismo se ocupa de los menos favorecidos, el principio de suficiencia sostiene que la justicia requiere que cada uno tenga la cantidad adecuada de los bienes especificados. ${ }^{6}$ Esta visión requiere que se satisfagan las necesidades, que previamente deben ser definidas en oposición a los deseos. ${ }^{7}$

El igualitarismo, el prioritarismo y el suficientarismo dan una especial importancia a los beneficios de los más desfavorecidos, por lo menos cuando se encuentran debajo del promedio o debajo del nivel adecuado. Esta prioridad no hace referencia en absoluto a lo que los individuos se merecen. ${ }^{5,8}$ Un acercamiento diferente a la justicia está interesado en asegurar que las personas obtengan lo que merecen.

Existe una variedad de visiones sobre las bases del merecimiento, es decir, ¿cómo determinar que una persona merece un beneficio? Unos pueden sostener que son las características de un individuo; otros, que su carácter o sus obras realizadas. No hay, por tanto, acuerdo sobre los elementos por considerar para recibir tal distinción. El mayor acuerdo se basa en el esfuerzo y la contribución a la sociedad como lo que podría distinguir a las personas. Se trata de una visión pragmática que sólo atiende a la productividad de una persona.

Una forma de aplicar la conjunción de las teorías sobre la distribución de recursos críticos para la atención de pacientes con COVID-19 sería: el acceso igual para todos en la atención primaria, para hacer la prueba y ser diagnosticados, el ser considerados para una 
Unidad de Cuidados Intensivos en caso de síntomas graves de la enfermedad como línea de suficiencia, dar prioridad a los pacientes por la probabilidad de éxito en caso de llegar al triage y el merecimiento sólo cuando se trate de personal de salud involucrado en la atención de estos mismos pacientes.

\section{TEORÍAS DE LA JUSTICIA}

Existen diversas teorías sobre la justicia según los diversos principios, reglas y juicios. ${ }^{2,9}$ Cada teoría intenta conectar las características de las personas con la distribución de beneficios y cargas moralmente justificables:

a) Las teorías utilitarias subrayan una mezcla de criterios con el propósito de asegurar al máximo la utilidad pública.

b) Las teorías liberales destacan los derechos de la libertad social y económica, recurriendo más a los procedimientos justos que a los resultados obtenidos.

c) Las teorías comunitarias hacen referencia a las prácticas de la justicia que se desarrollan en la tradición de una comunidad.

d) Las teorías igualitaristas que defienden el acceso igual a los bienes en la vida que toda persona racional valora (incluyendo criterios materiales de necesidad).

En esas condiciones, parece razonable partir de las teorías igualitaristas y comunitarias en el contexto de una nueva transformación social política de nuestro entorno, sin menospreciar las posturas utilitaristas y liberales que cuentan con los medios para alcanzar sus fines, siempre que sean justos y solidarios en la actualidad.

\section{LA PANDEMIA DE COVID-19 Y LOS CRITERIOS DE TRIAGE EN HOSPITALES SOBRECARGADOS}

La sociedad hoy reclama en la atención de la salud de pacientes enfermos por el coronavirus que haya justicia, equidad y transparencia en la asignación de recursos médicos. Ante la imposibilidad real de dar cuidados intensivos a todos los pacientes que los necesitan, surgen dilemas éticos de difícil solución. ${ }^{10}$

Una herramienta en situaciones de crisis en sistemas de salud saturados, como ha ocurrido en otros países, es la implementación del triage.
En esas condiciones y no otras, se entiende que se implementa el triage para procurar salvar la vida del mayor número de personas y en las mejores condiciones de supervivencia.

Lo ideal sería el poder evitar esa situación y tomar medidas para poder ofrecer cuidados intensivos a todos los pacientes que lo requieran, pero esa situación no ha sido viable ni en los países con mayor potencial económico, por las limitaciones de instalaciones de cuidados intensivos, los insumos y personal cualificado de medicina y enfermería crítica para dar esa atención. ${ }^{10}$

¿Cuáles son los puntos de controversia para asignar esos recursos limitados como los ventiladores en la pandemia o un lugar en las unidades de cuidados intensivos?

- La edad avanzada de un paciente es motivo para dejarlo en segundo plano.

- Si el paciente sufre una discapacidad que comprometa la supervivencia por enfermedades o trastornos previos.

- Si debe darse preferencia a los pacientes con mayor valía social.

\section{¿CUÁL DEBERÍA SER LA RESPUESTA BIOÉTICA A ESOS PLANTEAMIENTOS?}

La primera respuesta sería que ninguno de esos criterios debería ser considerado de forma aislada.

Siendo objetivos, los pacientes de mayor edad toleran menos la intubación y las posibilidades de recuperación son menores. Un ventilador podría pasar de ser un medio proporcionado a otro desproporcionado, cuando no diera el resultado esperado y eso no haría caer en la futilidad y/o la obstinación terapéutica.

Si el paciente tiene un deterioro cognitivo por una demencia senil, o padece de hipertensión, tiene diabetes o cuenta con infartos previos, sin redes de apoyo familiar, difícilmente podría sobrevivir a una enfermedad de esta naturaleza, buscando una supervivencia libre de discapacidad.

Ninguna discriminación es tolerable en el imaginario colectivo actual, pero se entiende que si se trata de personal de salud que en el ejercicio de su profesión en la atención de la pandemia contrajo la enfermedad, se convierte en recursos críticos para seguir operando el sistema de salud.

Con las consideraciones previas, se ha propuesto un algoritmo que incluya los diferentes elementos antes revisados, para que en los principales centros 
de salud se cuente con un equipo de triage que evalúe a los pacientes de forma periódica; de ser posible, cada día, para iniciar, continuar o retirar un tratamiento según los resultados obtenidos, con elementos tales como edad, saturación de oxígeno, diagnóstico previo de hipertensión arterial sistémica, elevación de enzimas cardiacas, conteo total de leucocitos, conteo de linfocitos y elevación de la creatinina sérica. En la práctica, lo que se evalúa específicamente en pacientes con COVID-19 son: gasometría, ferritina, dímero$\mathrm{D}$, procalcitonina, perfil hematológico, entre otros, para dar una idea de gravedad de la enfermedad sin que ello indique tampoco que los más graves tengan peor pronóstico.

Si estos criterios son tomados en cuenta como un modelo de justicia distributiva a cada uno, según su necesidad o cada uno según las posibilidades reales del sistema de salud, se evitaría la discrecionalidad en la toma de decisiones que el personal de salud puede implementar.

El tema de justicia distributiva ha sido aplicado para el trasplante de órganos, ya que se trata de un bien escaso con largas listas de espera, ${ }^{11,12}$ y puede ser la única esperanza de vida cuando un paciente tiene falla hepática o cardiaca; además, se sabe que es el tratamiento ideal en un paciente con insuficiencia renal crónica. En cada país, se ha tenido que discutir cuál es la mejor manera de asignar estos órganos y qué elementos se deben incluir en el sistema de puntaje cuando se emplea, para evitar la heterogeneidad de criterios en la toma de decisiones y su consecuente discrecionalidad. ${ }^{13}$

Ya Aristóteles decía: «Tan injusto es tratar a iguales desigualmente como a desiguales igualmente». Como ocurre en las situaciones de conflicto de interés, lo ideal sería poder evitarlas. Sin embargo, aunque la ética tiene siempre un fuerte componente aspiracional, nuestra indigencia ontológica nos hace recordar las limitaciones de espacio y tiempo con las que contamos como protagonistas precarios de la propia existencia.

Nadie duda del valor inestimable de cada vida humana, pero el ser humano continuamente está experimentando sus propias deficiencias: no lo podemos todo $\mathrm{ni}$ en el pensar ni en el querer ni en el hacer, tenemos una inteligencia creativa con una libertad finita y falible, por lo que es necesario formar equipos de trabajo para tomar las mejores decisiones e implementarlas.

Durante esta pandemia, más que nunca, la presencia de los cuidados paliativos es imperiosa. Dentro de los hospitales $\mathrm{y}$, en especial en las salas COVID, el trabajo de los médicos y enfermeras paliativistas son de gran soporte, tanto para el paciente como para la familia. No hay que olvidar a los psicólogos paliativistas, quienes hacen más llevadero el trabajo con el paciente y el personal de salud, quienes también requieren apoyo emocional y psicológico para enfrentar el día al día. ${ }^{14}$ Jamás tenemos que olvidar a la familia que se encuentra afuera, en casa, sin saber del estado del paciente, por lo que es de gran trascendencia la intervención del equipo paliativista para el apoyo de todos ellos, pues ellos hacen más llevadera la enfermedad, el aislamiento y la distancia. Desgraciadamente, no todos los hospitales de nuestro país cuentan con este servicio, pero es de suma importancia considerarlo en aquéllos donde sí los hay. Cuidados paliativos siempre se ha caracterizado por emplear la tecnología, hacer seguimiento, tratamiento por telemedicina y medicina a distancia, y en esta pandemia, no es la excepción; es algo que ha prevalecido y ayuda a unir a familiares y pacientes. ${ }^{15}$ Trabajando día con día con médicos de primer contacto, se puede apoyar a pacientes y familiares que no se encuentran en los hospitales, pero están en casa con uno o más familiares contagiados con COVID-19, y la presencia de la medicina paliativa apoya a la salud física, mental y espiritual del paciente y la familia. ${ }^{16}$

\section{GRUPOS VULNERABLES: PACIENTES TRASPLANTADOS}

No cabe duda que, dentro de los grupos vulnerables, a los que habría que priorizar para la atención del COVID-19 en las unidades de terapia intensiva son a los pacientes trasplantados. Son varios los motivos para proceder así:

- El trasplante de riñón, hígado o corazón ha representado una nueva oportunidad de vida.

- Para llevar acabo un trasplante, se ha realizado una gran inversión de recursos humanos, materiales y económicos que no es fácil evaluar, pero quienes trabajan en los programas, tanto de procuración como de trasplante, saben el gran esfuerzo que ha representado.

- Si, además, se trata de un niño o adolescente, se suma el criterio del interés superior del niño por la esperanza de vida que tiene.

- Por si fuera poco, otro motivo sería la enorme escasez de órganos para trasplantar como lo confirman las listas crecientes de pacientes en lista de espera. 
- La actividad de trasplante está suspendida temporalmente en nuestro país desde el 17 de marzo de 2020 por recomendación del Centro Nacional de Trasplantes (CENATRA). La gran mayoría de los centros de trasplante se reconvirtieron en centros COVID y, de momento, es riesgoso hacer el procedimiento. Por lo tanto, han aumentado las listas de espera y se asume que también los fallecimientos de pacientes en la espera de un órgano vital. Es de esperar que el SARS-CoV-2 se incluya como un virus más a estudiar en el binomio donador/receptor pretrasplante, y que deba vigilarse en el postrasplante, ya que por la inmunosupresión que reciben pueden no presentar todos los síntomas de caso sospechoso. ${ }^{17}$

Por todo lo anterior, queda más que claro que los pacientes trasplantados deberían ser prioridad en la atención de las Unidades de Terapia Intensiva. Sin embargo, aun con los argumentos aportados a favor de los receptores de trasplante, conocedores del ingente problema que atraviesan los sistemas de salud en todo el mundo, se pudiera esgrimir que esta población de pacientes ya tuvo su oportunidad en favor de la recuperación de la salud en el momento que pudieron recibir el beneficio del trasplante y que la situación de la pandemia afecta por igual a trasplantados y no trasplantados. En este sentido, sería mucho más legítimo, entonces, aplicar los mismos preceptos que han sido señalados en párrafos anteriores para la distribución de un recurso escaso que ha puesto al mundo entero «de cabeza», en la búsqueda del más justo equilibrio para la atención de todos.

\section{REFERENCIAS}

1. Brena Sesna I. El derecho y la salud. México: UNAM; 2004.

2. Rawls J. A theory of justice. Cambridge, MA: Harvard University Press; 1971.

3. Roemer JE. Equality of opportunity. Cambridge: Harvard University Press; 1998.

4. Smart J, Williams B. Utilitarianism: for and against. Cambridge: Cambridge University Press; 1973.
5. Parfit D. Equality or priority. In: Clayton M, Williams A. The ideal of equality. New York, NY: Palgrave Macmillan; 2001. pp. 81125.

6. Frankfurt H. Equality as a moral ideal. Ethics. 1987; 98: 21-43.

7. Casal P. Why sufficiency in not enough. Ethics. 2007; 117: 296326.

8. Arneson R. Luck egalitarianism and prioritarianism. Ethics. 2000; 110: 339-249.

9. Buchanan A. Justice as reciprocity versus subject-centered justice. Philosophy and Public Affairs. 1990; 19: 227-252.

10. Sprung CL, Joynt GM, Christian MD, Truog RD, Rello J, Nates JL. Adult ICU triage during the coronavirus disease 2019 pandemic: who will live and who will die? recommendations to improve survival. Crit Care Med. 2020.

11. Agich GJ. Extension of organ transplantation: some ethical considerations. Mt Sinai J Med. 2003, 70 (3): 141-147.

12. Perez-Tamayo R. The law, medical ethics, and transplants. Rev Invest Clin. 2005; 57 (2): 170-176.

13. Cantu-Quintanilla G, Alberu J, Reyes-Acevedo R, Medeiros M, Villa MS, Arreola JM et al. A comparative study of the traditional method, and a point-score system for allocation of deceaseddonor kidneys: a national multicenter study in Mexico. Transplant Proc. 2011; 43 (9): 3327-3330.

14. Tran DL, Lai SR, Salah RY, Wong AY, Bryon JN, McKenna MC et al. Rapid de-escalation and triaging patients in communitybased palliative care. J Pain Symptom Manage. 2020.

15. Committee to Evaluate Telemedicine for Aid-in-Dying Requests in the Context of the Coronavirus Epidemic. Telemedicine Policy Statement. Disponible en: https://www.psychiatry.org/ psychiatrists/covid-19-coronavirus/practice-guidance-forcovid-19

16. Recomendaciones para profesionales de atención domiciliaria en cuidados paliativos. Disponible en: https://cuidadospaliativos. org/blog/wp-content/uploads/2020/04/COMUNICADOSECPAL_AECPAL-SOBRE-RECOMENDACIONES-PARAEQUIPOS-DOMICILIARIOS-DE-CP.pdf

17. Kumar D, Manuel O, Natori Y, Egawa H, Grossi P, Han SH et al. COVID-19: a global transplant perspective on successfully navigating a pandemic. Am J Transplant. 2020. 